\title{
Numerical simulation of the cylindrical turbulent flow based on the pre-mixed abrasive jet mixing chamber
}

\author{
Dongsu Zhang 1, a, Yangkai Zhang ${ }^{1, b}$ \\ ${ }^{1}$ College of Mechanical Engineering, Anhui University of Science and \\ Technology, Huainan ,232001, China \\ a dszhang@aust.edu.cn, b2507312867@qq.com
}

\begin{abstract}
Keywords: mixing chamber; mixed effect; cylindrical turbulent; optimized design; numerical simulation.

Abstract. In this paper, the mixed abrasive jet mixing chamber was used as the research object, and the mixing efficiency was improved by introducing the flow around the cylinder in the mixing chamber to enhance the chaos of the flow and promote the turbulence fusion. The three parameters of cylindrical azimuth, feature size and axial position are designed and optimized by theoretical analysis, and the optimal scheme is given and the concrete implementation method is pointed out. In order to further verify the feasibility and practical effect of the scheme, this paper uses the computational fluid simulation software to simulate the mixing process in the two mixing chambers with or without cylindrical flow, and obtain the distribution cloud of the abrasive at the time of mixing. The comparison results show that the mixing effect of the mixing chamber is larger than that of the ordinary mixing chamber, which shows that the scheme is feasible and has some guiding effect on improving the mixing effect.
\end{abstract}

\section{Introduction}

In measuring the power of abrasive water jets, the mixing effect of abrasive and water flow is a crucial indicator. The quality of the mixing effect will directly determine the energy of the water jet. Therefore, in order to improve the mixing effect of abrasive and water flow, people put forward the pre-mixed abrasive jet based on the post-mixed abrasive jet. Although the pre-mixed and post-mixed, the mixing effect has been more substantial improvement, but still not ideal. The reason is that the mixing chamber which undertakes the main mixing function does not fuse well with the existing domestic, and international initiatives to improve the mixing effect such as improving the design of nozzles and pipes without solving the problems at the source[1].

Therefore, this paper discusses the mixing mechanism of abrasive and water flow, improves the existing mixing chamber and combines the mixing process in the mixing chamber so as to improve the mixing effect between abrasive and water flow, and enhances the mixing effect of the pre-mixed abrasive jet Provide a new idea.

\section{Physical and Mathematical Models of Abrasive Jet Mixing Chamber}

\section{The physical model of the mixing chamber}

The physical model of the pre-mixed abrasive jet mixing chamber is shown in Figure 1 . The high-pressure water flow enters from the right-hand inlet and the abrasive enters from the upper inlet under the action of the Gaza unit. Depending on the drag force between the water flow and the abrasive and the viscous drag Mixed, and finally through the left exit. 


\section{Mathematical model of force in mixed chamber}

Taking into account the abrasive particles in the water flow force is very complicated, to meet the actual needs of the project under the premise of considering only the main role of the water drag,

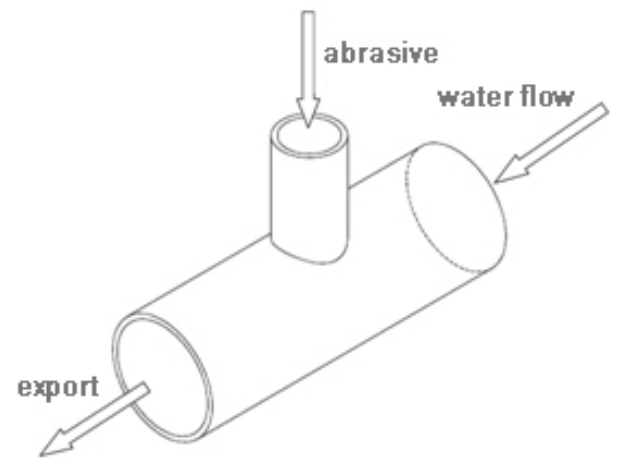

Fig.1 The physical model of the mixing chamber

abrasive gravity and other forces. According to Newton's second law can write the abrasive particle force differential equation [2].

$$
\frac{d u_{s}}{d t}=\frac{f}{\tau}\left(u-u_{s}\right)+\frac{g\left(\rho_{s}-\rho\right)}{\rho_{s}}+\frac{1}{2} \frac{\rho}{\rho_{s}} \frac{d}{d t}\left(u-u_{s}\right)
$$

Where $u$ and $u_{s}$ represent the velocity of the water flow and the abrasive particles, $\rho$ and $\rho_{s}$ represent the density of the water flow and the abrasive particles, $\tau$ represent the relaxation time of the particles, $\tau=\frac{\rho_{s} d_{s}{ }^{2}}{18 u}$ and $f$ represent the drag force function, $f=\frac{C_{D} R_{e s}}{24}$.

From eq.(1) shows that by increasing the drag function $\mathrm{f}$ method to improve the force of abrasive, thereby enhancing the mixing effect of water and abrasive.

Drag as the main force of the flow of abrasive, the greater the value, the higher the degree of chaos in the water flow, the more abrasive abrasive movement in the water more chaotic, the better the mixing effect [3]. In this paper, taking into account the actual situation of the mixing chamber, it is decided to introduce a cylindrical flow device in the mixing chamber to disturb the water flow and increase the chaos of the water flow.

\section{Cylindrical flow device design}

In this paper, the design of the cylinder around the flow device involved in the mixing chamber is divided into three parts, including the azimuth of the cylinder, the optimal size of the cylinder and the mixing chamber, and the axial position of the cylinder. The following three parts of the study, put forward their specific implementation plan.

\section{Azimuth of cylinder axis}

In view of the fact that there may be accumulation of abrasive and displacement in the actual mixing chamber, the sedimentation velocity of the particles should be less than the upward pulsation velocity [4] of the turbulent water in order to make the abrasive not sediment and accumulate in the water flow.

Azimuth $\alpha$ may take $0^{\circ}$ or $90^{\circ}$ to meet the processability of the designed mixing chamber. In order to make full use of velocity pulsation difference between the upper and lower layers after passing through the cylinder and the entrainment effect of the vortex on the abrasive, the vortices generated after flowing around should be located in a vertical plane. Therefore, in this paper, only azimuth $\alpha$ equals to $0^{\circ}$. 


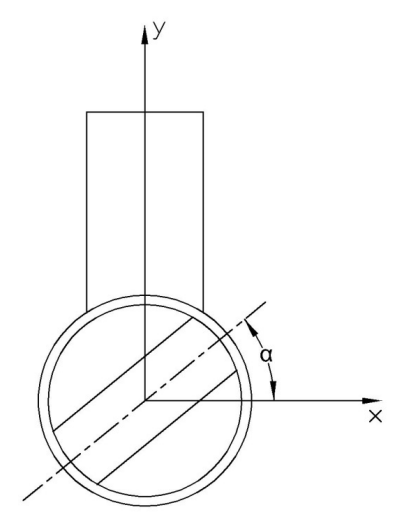

Fig. 2 Cylindrical azimuth

\section{Cylindrical and mixing chamber optimal size relationship}

In the past study of cylindrical flow around, the relationship between cylinder diameter and model size is often given directly based on empirical values, lift coefficient $C_{L}$ and $\operatorname{drag} \operatorname{coefficient} C_{D}$ as a fixed value. However, in this paper, in order to achieve the desired effect of mixing the abrasive in the wake, the lift coefficient $C_{L}$ should be as large as possible and the drag coefficient $C_{D}$ should be as small as possible. According to the literature [5], the lift coefficient $C_{L}$ and drag coefficient $C_{D}$ are defined as:

$$
\begin{aligned}
& C_{L}=\frac{2 F_{L}}{\rho U^{2} d H} \\
& C_{D}=\frac{2 F_{D}}{\rho U^{2} d H}
\end{aligned}
$$

In Eq. (2) and (3), $F_{L}$ represents the total lift, $F_{D}$ representing the total resistance.

Considering the actual shape of the mixing chamber, the ratio between cylinder diameter $d$ and mixing chamber diameter $D$ is approximately equal to the ratio of cylinder diameter $d$ to fluid calculation field height $H$ in the vertical direction, which can be approximated as $D$, which is defined as the diameter Than $L$.

$L=\frac{d}{H}$

Substituting Eq.(4) into Eq. (2) and Eq.(3) yields:

$$
\begin{aligned}
& C_{L}=\frac{2 F_{L}}{\rho U^{2} L H^{2}} \\
& C_{D}=\frac{2 F_{D}}{\rho U^{2} L H^{2}}
\end{aligned}
$$

By calculating the different diameter ratio of $C_{L}, C_{D}$ as shown in Table 1:

Table 1 lift coefficient of different diameters and drag coefficient

\begin{tabular}{cccccc}
\hline $\boldsymbol{L}$ & 0 & 0.1 & 0.2 & 0.3 & 0.4 \\
\hline $\boldsymbol{C}_{\mathbf{x}}$ & 0.15 & 0.26 & 0.37 & 0.42 & 0.41 \\
$\boldsymbol{c}_{\boldsymbol{D}}$ & 1.30 & 1.32 & 1.33 & 1.34 & 1.35 \\
$\boldsymbol{L}$ & 0.5 & 0.6 & 0.7 & 0.8 & 0.9 \\
$\boldsymbol{C}_{\mathbf{x}}$ & 0.39 & 0.36 & 0.32 & 0.28 & 0.24 \\
$\boldsymbol{C}_{\boldsymbol{D}}$ & 1.40 & 1.47 & 1.56 & 1.72 & 1.93 \\
\hline
\end{tabular}

As can be seen from Table 1 , when $L$ is in the range of 0.3 to 0.4 , the lift coefficient $C_{L}$ is the largest and the resistance coefficient $C_{D}$ is also ideal, indicating that the situation is the most superior. So in this article, $L$ takes 0.35 . 


\section{Cylindrical axial position}

The determination of the axial position of the cylinder should be based on its distance from the abrasive inlet $x$ to determine, in this paper, $x$ non-dimensional treatment, the definition of dimensionless parameter $t$ :

$t=\frac{x}{X}$

In equation (7), $X$ represents the total length of the mixing chamber.

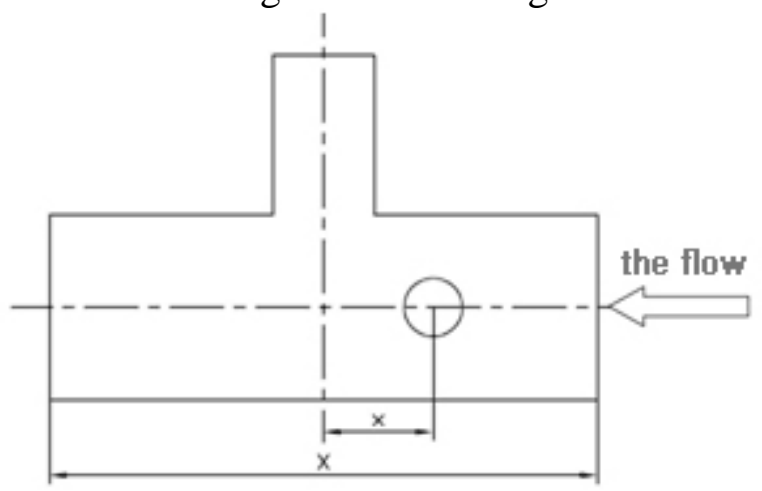

Fig.3 Cylindrical axial position

For best mixing between abrasive and incoming stream bypassing the wake, the abrasive should fall within the core of the wake and the turbulent mixing between the abrasive and the fluid is best, ie $t$ should exist reasonable value. To truly reflect the flow around the cylinder after the case of the trail, this paper uses a simulation, draw around the turbulent kinetic energy cloud image shown in Figure 4.

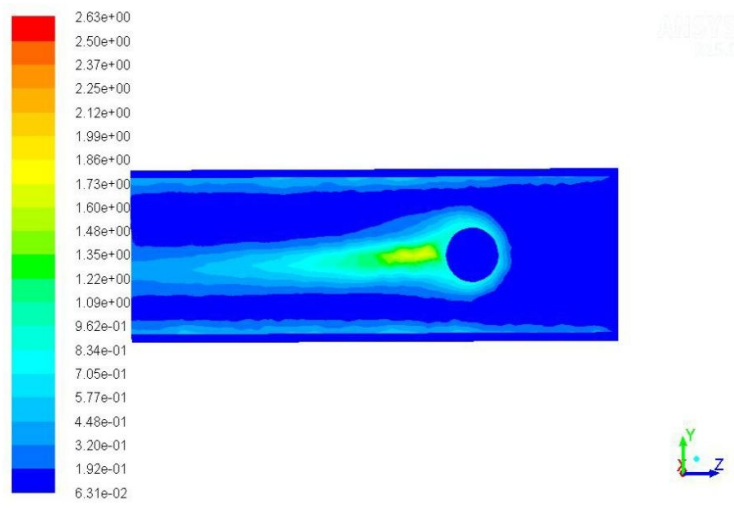

Fig.4 Turbulent kinetic energy flow chart

As can be seen from Figure 4, when $t$ is approximately equal to 0.2 , the abrasive is mixed in the core of the wake, the highest turbulent kinetic energy, from which the axial position of the cylinder can be determined.

At this point, the design of the cylinder around the flow device has been completed. The following combined with the actual production of the mixing chamber, the use of numerical simulation of the introduction of cylindrical flow around the device after the mixing effect for further discussion.

\section{Mixed-cavity simulation}

\section{Geometric model and meshing of mixing chamber}

Based on the mixing chamber used in the actual production, combined with the above design of the cylinder around the flow device, using Solidworks to create a solid model and import it into Fluent, add a fluid domain in $D M$ environment and define access. For a clearer view of the mixing between abrasive and water flow, the longitudinal section symmetry is defined considering the symmetrical distribution of the model. After the definition is completed, the fluid domain is meshed, and the result is shown in Figure 5: 


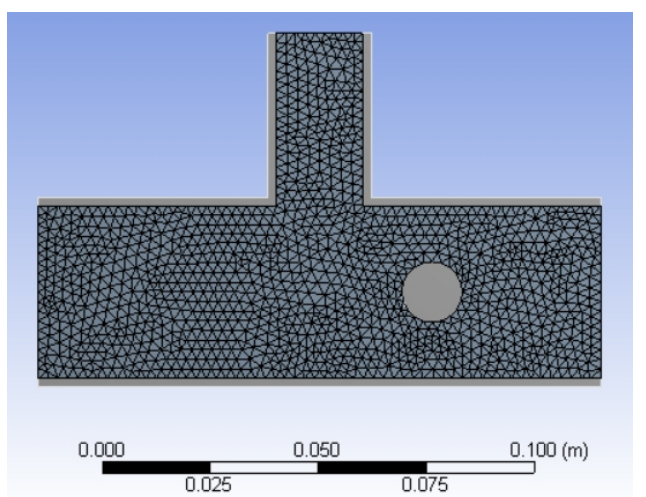

Fig. 5 Grid model

\section{Model establishment and solution calculation}

\section{The solve model}

Start a 3D solver using the Eulerian phase flow model and a standard 1 turbulence model. Their control equations are as follows:

(1) Euler multiphase flow control equations [6]

(1) momentum equation:

$\frac{\partial}{\partial t}\left(\rho_{m} v_{m}\right)+\nabla\left(\rho_{m} v_{m}{ }^{2}\right)=\nabla p+\nabla\left[\mu_{m}\left(\nabla v_{m}+\nabla v_{m}{ }^{T}\right)\right]+\rho_{m} g_{n}+F+\nabla\left(\sum_{i=1}^{n} \alpha_{i} \rho_{i} v_{d r}^{2}, i\right)$

Where $p$ is the isotropic pressure of the mixed fluid, $\mu_{m}$ is the viscosity of the mixed fluid, $T$ is the temperature of the mixed fluid, $F$ is the bulk force of the mixed fluid and $v_{d r}, i$ is the drift velocity of the i phase.

(2) second phase volume fraction equation:

$\frac{\partial}{\partial t}\left(\alpha_{2} \rho_{2}\right)+\nabla\left(\alpha_{2} \rho_{2} v_{m}\right)=-\nabla\left(\alpha_{2} \rho_{2} v_{d r}, i\right)$

Where $\alpha_{2}$ represents the volume fraction of the second phase.

(2) Standard $k-\varepsilon$ control equations [7]

(1) Time-averaged equation of motion:

$\frac{\partial\left(\rho u_{i}\right)}{\partial t}+\frac{\partial}{\partial x_{i}}\left(\rho u_{i} u_{j}\right)=\rho f_{i}-\frac{\partial p}{\partial x_{i}}+\frac{\partial}{\partial}\left[\mu\left(\frac{\partial u_{i}}{\partial x_{j}}+\frac{\partial u_{j}}{\partial x_{i}}\right)\right]-\frac{\partial}{\partial x_{j}}\left(\rho \overline{u_{i}^{\prime} u_{j}^{\prime}}\right)$

Where, $i, j$ is the coordinate component in the three-dimensional coordinate system, and $-\rho \overline{u_{i}^{\prime} u_{j}^{\prime}}$ is the Reynolds stress

(2)Turbulent energy $k$ equation:

$\rho \frac{\partial k}{\partial t}=\frac{\partial}{\partial x_{i}}\left[\left(\mu+\frac{\mu_{t}}{\sigma_{k}}\right) \frac{\partial \varepsilon}{\partial x_{i}}+G_{k}-\rho \varepsilon\right]$

Here, $k$ represents the kinetic energy of kinetic fluctuations per unit fluid and $\varepsilon$ represents the kinetic energy dissipation rate per unit fluid.

(3)Dissipation rate $\varepsilon$ equation:

$\rho \frac{\partial \varepsilon}{\partial t}=\frac{\partial}{\partial x_{i}}\left[\left(\mu+\frac{\mu_{t}}{\sigma_{\varepsilon}}\right) \frac{\partial \varepsilon}{\partial x_{i}}+C_{1 \varepsilon} \frac{\varepsilon}{k} G_{k}-C_{2 \varepsilon} \rho \frac{\varepsilon^{2}}{k}\right]$

Among them, $C_{1 \varepsilon}$ and $C_{2 \varepsilon}$ represent the constant coefficients, $\sigma_{k}$ and $\sigma_{\varepsilon}$ represent the $k-\varepsilon$ corresponding to a number of prandtl, and take 1.0 and 1.3 respectively in fluent [8,9].

\section{Boundary conditions}

Before setting the boundary conditions, the abrasive is considered as a continuous phase according to the fluidized condition of the abrasive. At the same time, the flow is defined as the basic phase and the abrasive is defined as the second phase. In this paper, the size of 80 mesh garnets, the density of $\rho_{s}=3800 \mathrm{~kg} / \mathrm{m}^{3}$ 
(1)Water inlet boundary conditions

For the continuous water phase, the inlet boundary condition is velocity-inlet. According to the water flow rate of $15 \mathrm{~L} / \mathrm{min}$ and the inlet diameter of $\varphi 5.2 \mathrm{~mm}$, the calculated inlet velocity is $11.7 \mathrm{~m} /$ s. At the same time, the turbulence intensity of the mixed phase is set to $5 \%$ and the hydraulic diameter is $\varphi 0.5 \mathrm{~mm}$. The volume fraction of the second phase is set to 0 . The remaining parameters remain the default.

(2) Abrasive inlet boundary conditions

For fluidized abrasive, the entrance boundary conditions are taken velocity-inlet . Set the inlet velocity of the abrasive to $2 \mathrm{~m} / \mathrm{s}$ and the volume percentage to 1 . The remaining parameters remain the default value unchanged.

(3) Other boundary conditions

For the near wall, this paper uses the standard wall function method. Other boundary conditions using the non-slip boundary conditions .

\section{The solve algorithm}

The SIMPLE algorithm is used to couple the pressure velocities, keeping the default convergence residuals and relaxation factors and initializing the fluid domain. After initialization, iterations are performed.

Using the same method, we simulate the two mixed cavities with and without a circular flow in turn. The following two mixed cavity mixing effect for comparison.

\section{Simulation results analysis}

By simulating the mixing process in the two mixing chambers, Volume Fraction cloud images of the abrasive in the longitudinal section of the two mixing chambers are obtained as shown in Fig. 6 and Fig. 7.

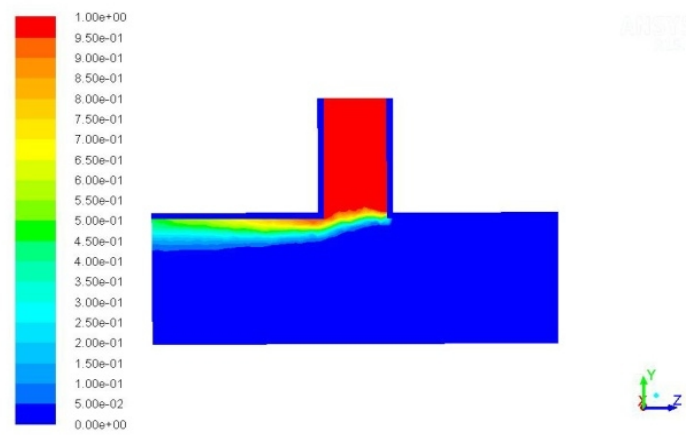

Fig. 6 Absence of a cylindrical flow around the mixing chamber in the abrasive distribution cloud

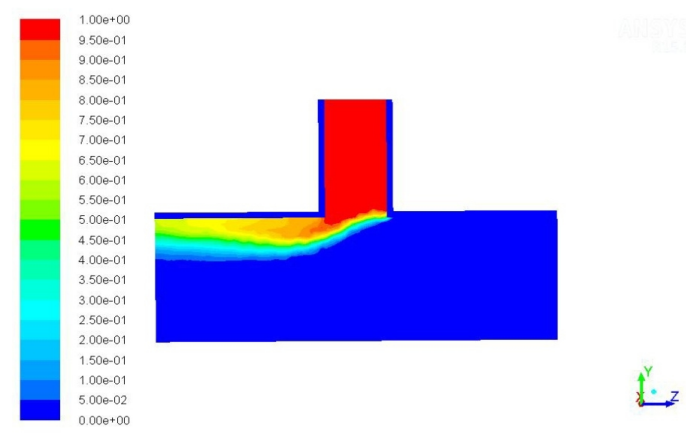

Fig. 7 Abrasive distribution cloud in the mixed flow around the cylinder

Comparing Fig. 6 and Fig. 7, we can see that in Fig. 7, after the flow around the cylinder, due to the formation of a wide range of turbulent fusion, the abrasive distributes more evenly in the water 
flow. After the abrasive enters the mixing chamber Of the mixed area has been greatly improved, which further validates the spoiler method for enhancing the mixing of abrasive is effective.

\section{Conclusion}

(1) The results of theoretical calculation and simulation show that it is an effective method to introduce a circular flow in the mixing chamber for the two-phase incompressible mixing layer of abrasive water in the mixing chamber.(2) The introduction of cylindrical flow, not only increases the degree of turbulence of the water flow, but also formed after the flow of large amplitude pulsation can promote turbulent fusion, thereby enhancing the mixing efficiency.(3) In order to optimize the mixing effect of abrasive and water flow, the diameter ratio between the cylinder and the mixing chamber and the dimensionless parameter for determining the axial position of the introduced cylindrical flow passing device have the optimal values. When the diameter ratio $\mathrm{L}=0.35$, the dimensionless parameter $t=0.2$ is the best solution, this time the most uniform mixing of abrasive and water.(4) The research in this paper provides a new idea for improving the mixing effect of the pre-mixed abrasive jet and has some guidance for actual production.(5) The following aspects can continue to be studied: to improve the quality of meshing to further improve the calculation accuracy; to quantify the mixing effect of abrasive and water, and to propose an effective quantitative method.

\section{Acknowledgements}

This work was financially supported by the Anhui Provincial Department of Education major research projects $(\mathrm{kj} 2016 \mathrm{SD} 18)$

\section{References}

[1] W.Q. Zuo, X.C. Wang, F.C.Hao, et al. Study on accelerating mechanism of abrasive grains based on iterative algorithm. Journal of China University of Petroleum (Natural Science Edition) .Vol.40 (2016): P.104-109

.[2] X. Dong, A.C. Ma, D.M. Li, numerical simulation of flow in DIA jet nozzle ,Mechanical Design and Research, Vol.21 (2005): P. 87-90.

[3] Z.H. Liu, C.L. Xu, Ho MUCHUAN, just like through-flow - reinforced flexible paddle mixer-settler tank oil composition - Water chaotic mixing, CHEMICAL TECHNOLOGY . Vol.68 (2017): P.637-642 .

[4] J.J. Sun, Water jet cutting technology. Xuzhou: China University of Mining and Technology Press, (1992)

[5] Y.L. Wang, Y.Z . Liu, G.P. Miu, Three Dimensional Numerical Simulation of Flow around a Cylinder .Journal of Shanghai Jiao tong University, Vol.35 (2001): P.1464-1469

[6] G.Y. Liang, Numerical simulation of two-phase flow of SK-type mixer based on ANSYS Workbench .Mail Mine Machinery, Vol.35 (2014): P. 63-64.

[7] T.C. Chen, Z.H. Guo, Y.Y. Lei, et al. Numerical simulation of flow field in abrasive water jet nozzles., Vol.10(2012): P. 66-68.

[8] W.Q. Tao, Numerical heat transfer. Xi'an: Xi'an Jiao tong University Press, (2001).

[9] C.C. Chen, W.W. Song, C.M. Yang, et al. Dimensional design of centrifugal, pump. Acta Automatic Machinery, Vol.33 (2002): P.34-36. 